\title{
The influence of resistance training on muscle strength, irisin concentration, and metabolic parameters in type 1 diabetic patients
}

\author{
Marta Wróbel ${ }^{1}$, Artur Gołaś2, Dominika Rokicka', tukasz Pyka ${ }^{3}$, Marta Szewczyk ${ }^{4}$, Tomasz Stołtny5, \\ Robert Roczniok², Mariusz Gąsior ${ }^{3}$, Krzysztof Strojek ${ }^{1}$ \\ ${ }^{1}$ Department of Internal Medicine, Diabetology, and Cardiometabolic Disorders, Faculty of Medical Sciences in Zabrze, Medical \\ University of Silesia, Katowice, Poland \\ ${ }^{2}$ Institute of Sport Sciences, The Jerzy Kukuczka Academy of Physical Education, Katowice, Poland \\ 33rd Department of Cardiology; Faculty of Medical Sciences in Zabrze, Medical University of Silesia, Katowice, Poland \\ ${ }^{4}$ Laboratory of Drug Monitoring, Silesian Centre for Heart Diseases in Zabrze, Zabrze, Poland \\ ${ }^{5}$ District Hospital of Orthopaedics and Trauma Surgery in Piekary Ślaskie, Piekary Ślaskie, Poland
}

\begin{abstract}
Introduction: Resistance effort has a beneficial effect on muscle mass, body composition, bone density, and cardiac parameters. It is also a modulator of the inflammatory reaction. The aim of the study was to assess the impact of 3 months of resistance training on muscle strength, irisin levels, and metabolic parameters in patients with long-term type 1 diabetes.

Material and methods: Eleven type 1 male diabetic patients with low levels of physical activity were recruited, with mean age $38 \pm 6$ years, body mass index (BMI) $28.4 \pm 2.6 \mathrm{~kg} / \mathrm{m}^{2}$, and diabetes duration $23 \pm 7$ years. All subjects participated in 60 -minute resistance training sessions twice a week, for three months. At baseline and after 3 months in all patients, maximal muscle strength level, serum irisin concentration, metabolic control parameters, and anthropometric measures were assessed.

Results: After 3 months there was a statistically significant increase of maximal muscle strength in comparison to baseline. There was no significant change in serum irisin concentration, $\mathrm{HbA}_{1 c^{\prime}}$ or other assessed parameters.

Conclusion: A 3-month resistance training programme in patients with long-term type 1 diabetes and low level of physical activity significantly affects their maximum strength level. This indicates that people with diabetes are more adaptive to additional loads, which allows them to increase their load faster. (Endokrynol Pol 2022; 73 (1): 96-102)
\end{abstract}

Key words: type 1 diabetes; resistance training; exercise; irisin

\section{Introduction}

Physical effort, along with proper nutrition, is the basis for behavioural treatment of diabetes. For many years aerobic exercise was the most recommended for diabetic patients, and resistance training was contraindicated. Currently, resistance training is treated as an important supplement to aerobic exercise in the therapy of diabetes [1].

Aerobic exercise is associated with an increased risk of hypoglycaemia in patients treated with insulin. Therefore, patients have to monitor their blood glucose levels frequently and consume additional carbohydrates during exercise. Conversely, patients performing resistance exercise have lower risk of hypoglycaemia, mainly due to elevated adrenaline levels that lead to the rapid release of glucose from the liver [2,3]. Resistance effort has a beneficial effect on muscle mass, body com- position, bone density, and cardiological parameters, including blood pressure and lipid profile $[4,5]$. It also increases insulin sensitivity. Most studies assessing the effects of exercise on the health status of patients with type 1 diabetes were conducted in young, physically active, or high-performance athletes [6-9]. Skeletal muscles play a mechanical role, are involved in energy metabolism, and support the immune system through anti-inflammatory action. Resistance training is known as an inflammatory response modulator. Skeletal muscles secrete proteins called "myokines" during exercise (over 100 myokines are known so far, including IL-1 receptor agonist, interleukin 10, and irisin), which have a beneficial effect on other tissues and reduce the concentration of some pro-inflammatory cytokines (e.g. TNF-alpha) [10]. In recent years, irisin has been of great interest; it is produced among other sources by skeletal muscle and is involved in the induction of the process of converting 
white adipose tissue (WAT) into brown adipose tissue (BAT). The precursor for this myokine is transmembrane protein FNDC5 (fibronectin type III domain containing $5)$, from which irisin is detached under the influence of a specific protease, which then enters the circulation [11]. It works by increasing expression in the uncoupling protein 1 (UCP-1) in mitochondria, and this leads to the conversion of white to brown fat tissue [12]. White adipose tissue is a supply of lipids for the body - it is rich in triglycerides and free fatty acids and has a low number of mitochondria. Due to the large number of mitochondria, brown adipose tissue plays a major role in energy expenditure and is responsible for thermogenesis [13]. Irisin seems to play a pleiotropic role especially in type 2 diabetes. Browning of white adipose tissue under the influence of irisin may be associated with beneficial effects of weight loss, on glucose and lipid homeostasis and endothelium. Irisin also acts as the inhibitor of lipogenesis and gluconeogenesis via the AMPK pathway, which has recently been shown in mice [14], and it might be a protective antidiabetic factor. It has been also shown that recombinant irisin has an antiapoptotic impact on pancreatic beta cells of humans and rodents — it stimulates their proliferation, insulin synthesis, and secretion. Its action seems to be similar to those of GLP-1 agonists (glucagon like peptide 1) and probably acts via special receptor for irisin, which has not yet been found on beta cells [15]. This myokine also has a positive impact on bone mineral density [16], which is often reduced in type 1 diabetic patients with long-lasting disease. Factors that affect irisin levels include physical activity, obesity, and diet [17]. There are not many scientific papers on the evaluation of irisin in adults with type 1 diabetes.

The increase in the level of muscle strength reflects the improvement in the level of neuromuscular coordination, better body awareness, and the economization and efficiency of the use of muscle groups in everyday life activities. Adaptation of the passive apparatus, elimination of the disproportion of muscle groups, and a better level of their synchronization should affect the control of diabetes (mean glycaemia, $\mathrm{HbA}_{1 \mathrm{c}}$ value, insulin demand) as well as anthropometric and laboratory parameters in physically inactive men with type 1 diabetes. The aim of the study was to assess the effect of 3 months of resistance training on muscle strength, irisin levels, and metabolic parameters in men with long-term type 1 diabetes.

\section{Material and methods}

\section{Patients}

The study included 11 men with long-term type 1 diabetes from Silesia Province (Poland), with low physical activity at baseline, and without advanced diabetes complications [age: $38 \pm 6$ years; body mass index (BMI) $28.4 \pm 2.6 \mathrm{~kg} / \mathrm{m}^{2}$; duration of diabetes $23 \pm$
7 years]. Patients were qualified for the study taking into account the following criteria:

- patients with type 1 diabetes aged 30-45 years, duration of diabetes at least 15 years;

- BMI 20-32 kg/m²

- treated with multiple insulin injections (at least 4/day) or a personal insulin pump;

- not physically active

- not planning to intensify effort during the study period;

- without significant valve defects in echocardiography, and with normal left ventricular ejection fraction (minimum 50\%);

- with a negative ECG exercise test,

- $\mathrm{HbA}_{1 \mathrm{c}}<8.5 \%$, without advanced diabetes complications (without albuminuria, overt autonomic neuropathy, more advanced than simple diabetic retinopathy).

Patients with advanced diabetes complications (e.g. no increase in heart rate during exercise), $\mathrm{HbA}_{1 c}>8.5 \%$, pre-proliferative and proliferative retinopathy, post-laser therapy, cardiovascular incident, coronary artery disease, microalbuminuria, and overt autonomic neuropathy were not qualified for the study.

The study was conducted in accordance with the Declaration of Helsinki, and the protocol was approved by the Ethics Committee at the Silesian Medical University in Katowice (KNW/0022/KB1/26/15). Written consent to participate in the study was obtained from all participants, after explaining the purpose of the study. All qualified patients underwent initial clinical tests, including cardiology, to assess their health before training.

\section{Methods}

Before starting training, the maximum load test (1RM) was performed for each exercise in each patient qualified for the study. The tests were carried out at the Laboratory of Muscle Strength and Power at the Jerzy Kukuczka Academy of Physical Education in Katowice. Maximum strength tests to determine the value of 1RM in 5 exercises (leg extension, lying leg curl, barbell upright row, wide pulldown, chest press) were performed a week before the first training session. Every testing and training session was performed using a HOIST Fitness System (California, USA). After performing a warm-up in accordance with the protocol [18], the subject conducted a series of maximum repetitions in such a way that the measure of maximum repetitions varied in the range 3-8 repetitions. Based on the value of one maximum repetition (1RM), the amount of external load with which the subjects performed 3 months of resistance training $(50 \% 1 \mathrm{RM})$ was calculated.

$1 R M=$ load $\times(1+0.0033 \times$ number of repetitions made $)$ [19]

The training unit consisted of 5 exercises involving most of the muscle groups (leg extension, lying leg curl, barbell upright row, wide pulldown, chest press). The subjects performed 5 sets of 10 repetitions of each of the resistance exercises. The interval between successive series and exercises was 90 seconds. The movement tempo was 2121 (2 seconds of eccentric and concentric work with a 1-second transition phase). The individual resistance training program was planned for 3 months, taking into account the basic principles of periodization of resistance training. Each week, the subject performed 2 training units (lasting 60 minutes). Progression of loads - both the number of series and the size of the external load - occurred weekly (min. 2\% $1 \mathrm{RM})$. After the end of the 3-month training period, a test protocol identical to the one before training was started.

Before starting the training program, all patients underwent the following: resting ECG, echocardiography, Holter ECG, Holter blood pressure, and fundus assessment. In addition, morphology, $\mathrm{HbA}_{1 c^{\prime}}$ concentration of aspartate transaminase and alanine transaminase, creatinine, urine albumin, and lipid profile were assessed. Baseline serum irisin were assessed in each study participant (irisin, fibronectin type III domain-containing protein 5 [FNDC5]) (test Irisin ELISA, BioVendor, Czech Republic). In addition, irisin levels were assessed within 1 hour immediately after the first training. 
Anthropometric tests were carried out: body weight, height, BMI, waist circumference, and body composition assessment (muscle mass, fat mass). Body weight and composition were evaluated in the morning hours (7-8 a.m.) after an overnight fast, using the electrical impedance method (Inbody 720, Biospace Co., Tokyo, Japan) before each phase of the experiment. Food and liquid intake was monitored the night before these measurements. The average daily insulin requirement and average daily glucose concentration (based on data from 1 week before start of training) were assessed. Glycaemia was monitored using the same model of glucometer for all patients (Contour Plus, Ascensia Diabetes Care, Germany). Patients committed to at least 6 measurements of glucose per day. Additional, required measurements on the training day were taken 15 minutes before training, after 30 minutes of training, and immediately after training. All patients had training on how to exercise during physical activity (matching insulin doses, counting carbohydrate exchanges, protein-fat exchanges).

The principles of dietary management during the training period were discussed with patients, with particular emphasis on the composition and regarding time of meal before and after training (patients also received it in writing). Each study participant was obligated to eat a carbohydrate-protein meal with fixed carbohydrate content (minimum 4-5 carbohydrate exchanges [40-50 grams of carbohydrates] + protein equivalent to $100 \mathrm{~g}$ of poultry meat). This meal was to be consumed no later than 1.5-2 hours before exercise to minimize the risk of hypoglycaemia. The subjects were also asked to consume a meal containing complex carbohydrates and protein (2-3 carbohydrate exchanges + protein [meat, fish, cheese]) within 1 hour after training. It was recommended that the pre-exercise target glucose range should be 140-180 mg/dL. After the third month of the training period, the following parameters were re-evaluated: morphology, $\mathrm{HbA}_{1 \mathrm{c}^{\prime}}$ concentration of aspartate transaminase and alanine transaminase, creatinine, urine albumin $(\mu \mathrm{g} / \mathrm{min})$, plasma irisin concentration, lipid profile, body weight, waist circumference, average daily insulin requirement (based on data from the last training week), average daily glucose concentration in the third month of the study [data from glucometers were read using the program GlucoContro Plus (Ascensia Diabetes Care, Germany)]. The frequency of hypoglycaemia in the $1^{\text {st }}$ and $3^{\text {rd }}$ (last) month of the study was also evaluated.

\section{Statistical analysis}

In order to characterize the structure of the studied variables, means and standard deviations were calculated. The Shapiro-Wilk test was used to verify the normality of the distribution of the analysed variables. To verify the significance of differences before and after the training, significance tests of dependent samples were used. If the variables had normal distributions, Student's t-test was used for dependent samples; after finding extreme asymmetries, the Wilcoxon pair order test was used. All analyses were performed using the Statistica v.13 package. A significance level of 0.05 was used for all analyses. Regarding measurements for all variables, the intraclass correlation coefficient (ICC) was high (0.96-0.99).

\section{Results}

Table 1 presents the general characteristics of the group as well as the parameters of somatic and biochemical structure, insulin demand, and the frequency of hypoglycaemia before and after training. The tested parameters did not change at the end of training sessions.

\section{Analysis of maximum strength levels}

The analysis of the level of muscle strength showed a statistically significant increase in the level of maxi- mum strength in participants in each of the exercises after a 3-month training period (leg extension, lying leg curl, barbell upright row, wide pulldown, chest press) $(\mathrm{p}<0.05$, Tab. 2).

\section{Irisin}

We observed a comparable baseline irisin concentration among the patients of our study in relation to the control group, who were healthy men, physically inactive, and at a comparable age (9.85 \pm 4.7 vs. $10.3 \pm 2.1 \mu \mathrm{g} / \mathrm{mL}$ ). A statistically insignificant decrease in irisin concentration immediately after the first training $(8.16 \pm 3.7 \mu \mathrm{g} / \mathrm{mL}, \mathrm{p}=0.2)$ and after 3 months of training $(8.4 \pm 2.0 \mu \mathrm{g} / \mathrm{mL}, \mathrm{p}=0.37)$ was found, compared to the initial value.

\section{Safety}

No side effects were recorded during the study.

\section{Discussion}

Our results indicate that a 3-month period of resistance training in patients with long-term type 1 diabetes, at the beginning physically inactive, significantly affects their maximal strength level (1RM value). The increase in the level of muscle strength obtained by the participants of our study is much larger than with similar muscle strength training procedures used in healthy beginners [20]. The results of strength training are very well-described in the literature; relying on this fact, we decided not to involve a control group. According to the literature reports, in healthy subjects the increase in the level of muscle strength over a period of 3 months ranges from $30 \%$ to $50 \% 1 \mathrm{RM}$ [21-23]. In our study the maximal strength level increased between 70-100\% $1 R M$ for the specific variables after 3 months (Tab. 2). It was not observed that the applied resistance training had an effect on plasma irisin concentration, metabolic control parameters, lipid profile, or anthropometric parameters.

The significant improvement in muscle strength achieved after a 3-month training period is probably because the study participants are insulin-treated patients. Insulin works synergistically with growth hormone and with IGF-1. It is an anabolic hormone; hence, it is used as a doping agent by healthy people practicing in sports at a competitive level [24]. Insulin increases protein synthesis in muscle, fat, and liver tissue and inhibits their breakdown. In addition, it facilitates the transport of glucose and amino acids into the cell and increases the rate of glycolysis by increasing the activity of hexokinase and 6-phospho-kinase [25]. Insulin increases glycogen synthesis and accumulation during the restitution period and inhibits its breakdown, and it 
Table 1. General characteristics, parameters of somatic structure, and biochemical parameters before and after muscle strength training

\begin{tabular}{|c|c|c|c|c|c|}
\hline \multirow{2}{*}{ Variables } & \multicolumn{2}{|c|}{ Baseline } & \multicolumn{2}{|c|}{ After 3 months } & \multirow[t]{2}{*}{$\mathbf{p}$} \\
\hline & Mean & SD & Mean & SD & \\
\hline Age (years) & 35 & 6 & & & \\
\hline Diabetes duration [years] & 23 & 7 & & & \\
\hline Urine albumin $[\mu \mathrm{g} / \mathrm{min}]$ & 4.70 & 5.24 & 4.97 & 5.34 & 0.22 \\
\hline $\mathrm{HbA}_{1 \mathrm{c}}(\%)$ & 7.36 & 0.98 & 7.28 & 0.79 & 0.16 \\
\hline $\mathrm{ALT}[\mathrm{U} / \mathrm{L}]$ & 26.73 & 19.48 & 33.36 & 26.53 & 0.40 \\
\hline AST [U/L] & 22.73 & 6.94 & 27.91 & 18.11 & 0.39 \\
\hline Creatinine $[\mu \mathrm{mol} / \mathrm{L}]$ & 74.27 & 11.65 & 75.55 & 11.78 & 0.47 \\
\hline Total cholesterol [mmol/L] & 5.34 & 1.09 & 5.29 & 0.79 & 0.87 \\
\hline HDL-cholesterol [mmol/L] & 1.50 & 0.40 & 1.51 & 0.37 & 0.92 \\
\hline LDL-cholesterol [mmol/L] & 3.11 & 1.05 & 3.23 & 0.84 & 0.87 \\
\hline Triglycerides $[\mathrm{mmol} / \mathrm{L}]$ & 1.39 & 0.75 & 1.23 & 0.85 & 0.57 \\
\hline Body mass $[\mathrm{kg}]$ & 91.68 & 9.77 & 91.88 & 9.64 & 0.61 \\
\hline Muscle mass [kg] & 37.74 & 4.19 & 38.46 & 4.36 & 0.17 \\
\hline Fat mass $[\mathrm{kg}]$ & 24.79 & 7.36 & 23.97 & 8.45 & 0.26 \\
\hline$\%$ fat & 26.78 & 6.22 & 25.73 & 7.58 & 0.21 \\
\hline BMI $\left[\mathrm{kg} / \mathrm{m}^{2}\right]$ & 28.41 & 2.66 & 28.42 & 2.70 & 0.94 \\
\hline Waist circumference [cm] & 101.45 & 8.36 & 100.36 & 8.59 & 0.26 \\
\hline Insulin — total daily dose (U/24 h) & 72 & 20.4 & 67 & 16.8 & 0.09 \\
\hline Insulin/kg body mass & 0.78 & 0.17 & 0.73 & 1.14 & 0.08 \\
\hline Mean glucose $[\mathrm{mg} / \mathrm{dL}]$ & 162 & 32.2 & 168 & 19.0 & 0.39 \\
\hline $\begin{array}{l}\text { Hypoglycaemia (below 70/mg/dL), } \\
\text { amount of episodes/month }\end{array}$ & $\begin{array}{c}16 \\
\text { (1 month) }\end{array}$ & 11 & $\begin{array}{c}11 \\
\text { (3 month) }\end{array}$ & 9 & 0.27 \\
\hline
\end{tabular}

$\mathrm{HbA}_{1 \mathrm{c}}$ — haemoglobin $\mathrm{A}_{1 \mathrm{c}} ; \mathrm{LDL}$ — low-density lipoprotein; $\mathrm{HDL}$ — high-density lipoprotein; ALT — alanine transaminase; AST — aspartate transaminase; $\mathrm{BMI}$ - body mass index; $\mathrm{SD}$ - standard deviation

Table 2. Analysis of maximum strength level (1RM) in the applied resistance exercises before and after 3 months of muscle strength training. Data are presented as mean and standard deviation (SD)

\begin{tabular}{lccccc}
\hline \multirow{2}{*}{ Variables } & \multicolumn{2}{c}{ Baseline } & \multicolumn{2}{c}{ After 3 months } & \multirow{2}{*}{ p } \\
\cline { 2 - 5 } & Mean & SD & Mean & SD & $<0.00001$ \\
\hline Leg extension, 1RM $[\mathrm{kg}]$ & 41.72 & 6.83 & 71.88 & 7.63 & $<0.00001$ \\
\hline Lying leg curl, 1RM $[\mathrm{kg}]$ & 36.59 & 3.40 & 62.27 & 3.44 & 0.003 \\
\hline Barbell upright row, 1RM $[\mathrm{kg}]$ & 37.70 & 3.01 & 67.50 & 2.24 & $<0.00001$ \\
\hline Wide pulldown, 1RM $[\mathrm{kg}]$ & 44.09 & 7.10 & 88.41 & 7.52 & $<0.00001$ \\
\hline Chest press, 1RM $[\mathrm{kg}]$ & 36.36 & 6.16 & 70.41 & 10.87 & $<$ \\
\hline
\end{tabular}

SD — standard deviation

accelerates muscle regeneration after exercise. In addition, it causes hypertrophy of muscles and an increase in their strength due to a much greater amount of accumulated glycogen after exercise compared to that seen in people without diabetes [26]. According to the results of studies from the 1980s, a type 1 diabetic patient has a lower ability to accumulate hepatic glycogen stores compared to healthy people, which could be related to insufficient glycaemic control in patients as a result of the unavailability of intensive insulin therapy at the time [27]. However, it has been shown that patients with type 1 diabetes, metabolically well controlled, have comparable hepatic and muscle glycogen stores assessed by magnetic resonance imaging $\left({ }^{13} \mathrm{C}\right.$-magnetic resonance spectroscopy [MRS]) with healthy individuals [28]. Participants in our study were metabolically 


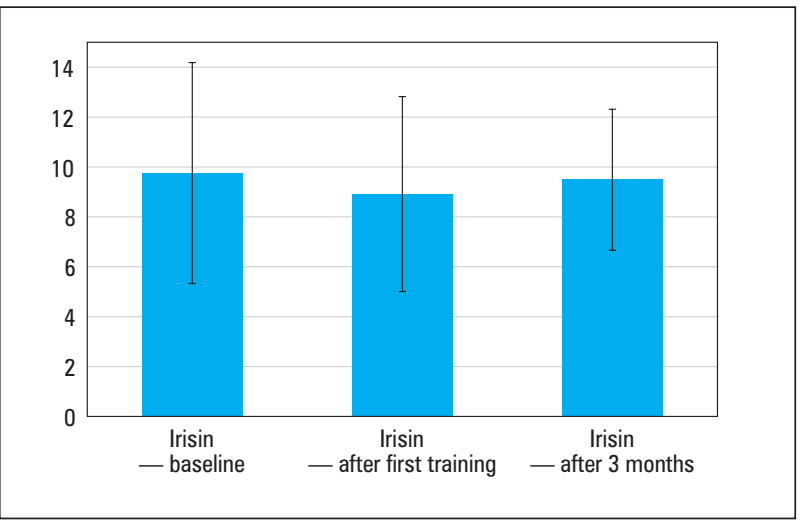

Figure. 1. Irisin concentration $[\mu \mathrm{g} / \mathrm{mL}]$ mean and standard deviation (SD): baseline, directly after 1st training, and after 3 months

well controlled $\left(\mathrm{HbA}_{1 \mathrm{c}} 7.3 \%\right)$. Therefore, we assume that the initial muscle glycogen supply was comparable to that in healthy people. The increase in maximum strength obtained in the study shows that people with type 1 diabetes are more adaptable to additional loads, and this allows them to progress faster.

Despite the lack of a statistically significant improvement in glycaemic control, a downward trend in $\mathrm{HbA}_{1 c}$ value was observed. Our participants had initially well-controlled diabetes; therefore, a spectacular reduction of $\mathrm{HbA}_{1 c}$ should not be expected. The glucose value, both during and after exercise, results not only from its intensity and duration, but also from several other factors such as time of day, baseline glycaemia, amount of active insulin injected before last meal, type of meal consumed, type and amount of carbohydrates taken during exercise, the patient's knowledge of managing disease in practice, etc. The level of knowledge of the participants of our study was considered as high.

In patients with type 1 diabetes, insulin levels will not decrease when they start exercising (the subcutaneous hormone will work in accordance with its pharmacokinetics), so these patients are at particular risk of hypoglycaemia. Resistance training, in contrast to moderate oxygen training, allows for a more stable glycaemic profile, and in some people it even results in an increase in glucose requiring a correction dose of insulin [3]. In this case, hypoglycaemia is less common than with moderate aerobic exercise. The lower frequency of hypoglycaemia (not statistically significant) in the third month of the study compared to the frequency of hypoglycaemia in the first month was probably the result of a gradually increased intensity of resistance training, and thus more pronounced hepatic glucose production under the influence of secreted counter-regulatory hormones. Serious hypoglycaemia, defined as requiring the help of a second person, was not noted in our study. The participants of our study were middle-aged ( 35 years old), overweight $(\mathrm{BMI}=28)$, leading mainly a sedentary lifestyle. This is a representative sample of the type 1 diabetes population because according to recent data overweight or obesity is found in approximately $60 \%$ of adult patients with this type of disease [29]. The fact that our patients did not reduce their body weight after a 3-month training period does not mean that exercise was not suitable for them. The purpose of the used resistance training was not to reduce body fat, which is justified by exercise physiology. In resistance training, free fatty acids are not the main "fuel", in contrast to moderate aerobic exercise, which is therefore recommended for weight reduction.

In our study, the effect of resistance exercise on the concentration of irisin - a myokine produced both by skeletal muscle and in small amounts by adipose tissue - was also analysed. The main factor responsible for the synthesis of irisin is physical exertion. It is a pleiotropic hormone that not only has a beneficial effect on glucose and lipid metabolism, but also stimulates myogenesis of skeletal muscles, inhibiting their atrophy and protecting against bone loss. It has been shown that increased irisin levels in patients with type 1 diabetes are associated with improved bone mass, which is particularly important because with prolongation of diabetes the risk of osteoporosis increases [30].

There are not many scientific papers on irisin in adults with type 1 diabetes [31, 32]. Hence, there is a lack of consistent data on the concentration of this myokine compared to healthy people [32, 33]. There are reports that healthy, physically active people have higher irisin values compared to inactive people [34]. In our study, the baseline plasma irisin concentration was comparable to the control group. According to recent reports, irisin has been found in pancreatic cells located peripherally to Langerhans islets [11]. In one study conducted in adults with type 1 diabetes, lasting 3 years on average, a higher concentration of irisin compared to healthy people was found. Moreover, irisin levels were higher in the group of people with anti-glutamic acid decarboxylase (anti-GAD) and anti-islet cell (anti-ICA) antibodies. Researchers explain this fact by the increased release of irisin due to the presence of chronic low-grade inflammation within the pancreas [35]. Perhaps with the duration of type 1 diabetes, the irisin concentration decreases, and therefore in our study participants, with 23 years of illness, no difference compared to healthy people was found. Similarly, in another study, lower irisin levels were observed in people who developed type 1 diabetes in early childhood [32]. The baseline lack of differences between irisin levels compared to the control group 
may also be due to the low baseline level of training for both our study participants and healthy volunteers in the control group.

Irisin is produced in response to physical exertion. The highest concentration is reached between 3 and 60 minutes after exercise; it returns to baseline after 6 hours [35]. To assess short-term changes in plasma irisin concentrations, we collected blood from patients within 1 hour of the first training. Our lack of growth could have been due to the fact that the first training was associated with the lowest muscle load. We also did not observe statistically significant changes in irisin concentration after the end of the 3-month training period from baseline. Data on the impact of the type of exercise and its intensity on irisin levels are not entirely clear, even in healthy people. Some studies have shown that resistance effort and endurance training are associated with increased irisin levels [17]. On the other hand, prolonged physical effort and aerobic effort do not affect the concentration of this parameter [36]. In a cross-sectional study evaluating adolescents with type 1 diabetes, the highest levels of this parameter were found in people who undertook intense physical activity ( 3 times a week for at least 60 minutes) [34].

Therefore, it is difficult to relate the results obtained by us to the currently available studies, in which irisin concentration was assessed in response to a different type of physical effort. Perhaps other previously unknown factors affect the concentration of exercise-induced irisin and its effect on other tissues. Nevertheless, due to the wide range of suggested beneficial effects (effects on carbohydrate and lipid metabolism, neuron and osteoblast differentiation, etc.), besides fulfilling the regulatory function in communication between active muscle tissue and adipose tissue and other organs, irisin requires further research in patients with diabetes type 1 .

\section{Conclusions}

The significant increase in the maximum strength level after resistance training in type 1 diabetic patients indicates that they are more adaptable to additional loads, and it allows them to increase the load faster, in comparison to healthy people. Seeing the effect of their work, patients feel motivated to continue training and change their lifestyle. Therefore, they should be able to individually choose the type of training in accordance with their own needs. Restricting the patient at the outset by prohibiting, for example, strength exercises, may discourage them from undertaking any physical activity.

\section{Limitations}

The limitations of the study are primarily the lack of control group, a small number of patients, and a short observation period.

\section{Conflict of interests}

The authors declare no conflicts of interest

\section{References}

1. American Diabetes Association. Standards of Medical Care in Diabetes - 2020. Diab. Care. 2020; 43(Suppl 1): 48-65, doi: 10.2337/diacare.28. suppl_1.s4, indexed in Pubmed: https://doi.org/10.2337/dc20-Sint.

2. Frayn KN, Karpe F, Funada Ji, et al. Subcutaneous adipose tissue blood flow varies between superior and inferior levels of the anterior abdominal wall. Int J Obes Relat Metab Disord. 2004; 28(2): 228-233, doi: 10.1038/sj.ijo.0802541, indexed in Pubmed: 14647178.

3. Riddell MC, Gallen IW, Smart CE, et al. Exercise management in type 1 diabetes: a consensus statement. Lancet Diabetes Endocrinol. 2017; 5(5): 377-390, doi: 10.1016/S2213-8587(17)30014-1, indexed in Pubmed: 28126459

4. Garber CE, Blissmer B, Deschenes MR, et al. American College of Sports Medicine. American College of Sports Medicine position stand. Quantity and quality of exercise for developing and maintaining cardiorespiratory, musculoskeletal, and neuromotor fitness in apparently healthy adults: guidance for prescribing exercise. Med Sci Sports Exerc. 2011; 43(7): 1334-1359, doi: 10.1249/MSS.0b013e318213fefb, indexed in Pubmed: 21694556

5. Quirk H, Blake H, Tennyson R, et al. Physical activity interventions in children and young people with Type 1 diabetes mellitus: a systematic review with meta-analysis. Diabet Med. 2014; 31(10): 1163-1173, doi: 10.1111/dme.12531, indexed in Pubmed: 24965376.

6. Laaksonen DE, Atalay M, Niskanen LK, et al. Aerobic exercise and the lipid profile in type 1 diabetic men: a randomized controlled trial. Med Sci Sports Exerc. 2000; 32(9): 1541-1548, doi: 10.1097/00005768-2000090 00-00003, indexed in Pubmed: 10994902.

7. Landt KW, Campaigne BN, James FW, et al. Effects of exercise training on insulin sensitivity in adolescents with type I diabetes. Diabetes Care. 1985; 8(5): 461-465, doi: 10.2337/diacare.8.5.461, indexed in Pubmed: 4053932.

8. Yardley JE, Kenny GP, Perkins BA, et al. Effects of performing resistance exercise before versus after aerobic exercise on glycemia in type 1 diabetes. Diabetes Care. 2012; 35(4): 669-675, doi: 10.2337/dc11-1844, indexed in Pubmed: 22374639.

9. Yardley JE, Kenny GP, Perkins BA, et al. Effects of performing resistance exercise before versus after aerobic exercise on glycemia in type 1 diabetes. Diabetes Care. 2012; 35(4): 669-675, doi: 10.2337/dc11-1844, indexed in Pubmed: 22374639.

10. Gleeson M, Bishop NC, Stensel DJ, et al. The anti-inflammatory effects of exercise: mechanisms and implications for the prevention and treatmen of disease. Nat Rev Immunol. 2011; 11(9): 607-615, doi: 10.1038/nri3041, indexed in Pubmed: 21818123

11. Aydin S, Kuloglu T, Aydin S, et al. A comprehensive immunohistochemical examination of the distribution of the fat-burning protein irisin in biological tissues. Peptides. 2014; 61: 130-136, doi: 10.1016/j. peptides.2014.09.014, indexed in Pubmed: 25261800.

12. Rabiee F, Lachinani L, Ghaedi $\mathrm{S}$, et al. New insights into the cellula activities of Fndc5/Irisin and its signaling pathways. Cell Biosci. 2020; 10: 51, doi: 10.1186/s13578-020-00413-3, indexed in Pubmed: 32257109.

13. Enerbäck S. Human brown adipose tissue. Cell Metab. 2010; 11(4): 248-252, doi: 10.1016/j.cmet.2010.03.008, indexed in Pubmed: 20374955

14. Mo Li, Shen J, Liu Q, et al. Irisin Is Regulated by CAR in Liver and Is a Mediator of Hepatic Glucose and Lipid Metabolism. Mol Endocrinol. 2016; 30(5): 533-542, doi: 10.1210/me.2015-1292, indexed in Pubmed: 27007446.

15. Natalicchio A, Marrano N, Biondi G, et al. The Myokine Irisin Is Released in Response to Saturated Fatty Acids and Promotes Pancreatic $\beta$-Cell Survival and Insulin Secretion. Diabetes. 2017; 66(11): 2849-2856, doi: 10.2337/db17-0002, indexed in Pubmed: 28724742

16. Colaianni G, Cuscito C, Mongelli T, et al. The myokine irisin increases cortical bone mass. Proc Natl Acad Sci U S A. 2015; 112(39): 12157-12162 doi: 10.1073/pnas.1516622112, indexed in Pubmed: 26374841.

17. Mahgoub MO, D'Souza C, Al Darmaki RS, et al. An update on the role of irisin in the regulation of endocrine and metabolic functions. Peptides. 2018; 104: 15-23, doi: 10.1016/j.peptides.2018.03.018, indexed in Pubmed: 29608940 
18. Gołaś A Maszczyk A, Pietraszewski P, et al. Effects of Pre-exhaustion on the Patterns of Muscular Activity in the Flat Bench Press. J Strength Cond Res. 2017; 31(7): 1919-1924, doi: 10.1519/JSC.0000000000001755, indexed in Pubmed: 27984499.

19. Baechle TR, Earle RW. Essential of strength training and conditioning. Human Kinetics, Champaing 2008

20. Stastny P, Gołaś A, Blazek D, et al. A systematic review of surface electromyography analyses of the bench press movement task. PLoS One. 2017; 12(2): e0171632, doi: 10.1371/journal.pone.0171632, indexed in Pubmed: 28170449.

21. Aaberg E. Muscle mechanics. 2nd ed. Human Kinetics, Library of Congress Cataloging-in-Publication Data, Champaign 2016: Second.

22. Flanagan SD, Mills MD, Sterczala AJ, et al. The relationship between muscle action and repetition maximum on the squat and bench press in men and women. J Strength Cond Res. 2014; 28(9): 2437-2442, doi: 10.1519/JSC.0000000000000337, indexed in Pubmed: 24343331.

23. Fleck SJ, Kreamer WJ. Designing Resistance Training Programs. 4th ed. Human Kinetics, Champaign 2014.

24. Holt RIG, Sönksen PH. Growth hormone, IGF-I and insulin and their abuse in sport. Br J Pharmacol. 2008; 154(3): 542-556, doi: 10.1038/bjp.2008.99, indexed in Pubmed: 18376417.

25. Kahn CR, Weir GC, King GL. Joslin's Diabetes Mellitus. 14th ed. Lippincott Williams and Wilkins, Philadelphia 2007: 649-657.

26. Fink J, Schoenfeld BJ, Nakazato K. The role of hormones in muscle hypertrophy. Phys Sportsmed. 2018; 46(1): 129-134, doi: 10.1080/00913 847.2018.1406778, indexed in Pubmed: 29172848.

27. Hwang JH, Perseghin G, Rothman DL, et al. Impaired net hepatic glycogen synthesis in insulin-dependent diabetic subjects during mixed meal ingestion. A 13C nuclear magnetic resonance spectroscopy study. J Clin Invest. 1995; 95(2): 783-787, doi: 10.1172/JCI117727, indexed in Pubmed: 7860761.

28. Bally L, Buehler T, Dokumaci AS, et al. Hepatic and intramyocellular glycogen stores in adults with type 1 diabetes and healthy controls. Diabetes
Res Clin Pract. 2015; 109(1): e1-e3, doi: 10.1016/j.diabres.2015.05.002, indexed in Pubmed: 26013568.

29. Bohn B, Herbst A, Pfeifer M, et al. DPV Initiative. Impact of Physical Activity on Glycemic Control and Prevalence of Cardiovascular Risk Factors in Adults With Type 1 Diabetes: A Cross-sectional Multicenter Study of 18,028 Patients. Diabetes Care. 2015; 38(8): 1536-1543, doi: 10.2337/dc15-0030, indexed in Pubmed: 26015557.

30. Faienza MF, Brunetti G, Sanesi L, et al. High irisin levels are associated with better glycemic control and bone health in children with Type 1 diabetes. Diabetes Res Clin Pract. 2018; 141: 10-17, doi: 10.1016/j.diabres.2018.03.046, indexed in Pubmed: 29679630.

31. Ates I, Arikan MF, Erdogan K, et al. Factors associated with increased irisin levels in the type 1 diabetes mellitus. Endocr Regul. 2017; 51(1): 1-7, doi: 10.1515/enr-2017-0001, indexed in Pubmed: 28222023.

32. Espes D, Lau J, Carlsson PO. Increased levels of irisin in people with long-standing Type 1 diabetes. Diabet Med. 2015; 32(9): 1172-1176, doi: 10.1111/dme.12731, indexed in Pubmed: 25762196.

33. Tentolouris A, Eleftheriadou I, Tsilingiris D, et al. Plasma Irisin Levels in Subjects with Type 1 Diabetes: Comparison with Healthy Controls. Horm Metab Res. 2018; 50(11): 803-810, doi: 10.1055/a-0748-6170, indexed in Pubmed: 30286484.

34. Morelli C, Avolio E, Galluccio A, et al. Impact of Vigorous-Intensity Physical Activity on Body Composition Parameters, Lipid Profile Markers, and Irisin Levels in Adolescents: A Cross-Sectional Study. Nutrients. 2020; 12(3), doi: 10.3390/nu12030742, indexed in Pubmed: 32168929.

35. Nygaard H, Slettaløkken G, Vegge G, et al. Irisin in blood increases transiently after single sessions of intense endurance exercise and heavy strength training. PLoS One. 2015; 10(3): e0121367, doi: 10.1371/journal. pone.0121367, indexed in Pubmed: 25781950.

36. Peterson JM, Mart R, Bond CE. Effect of obesity and exercise on the expression of the novel myokines, Myonectin and Fibronectin type III domain containing 5. PeerJ. 2014; 2: e605, doi: 10.7717/peerj.605, indexed in Pubmed: 25289190. 